\title{
The ubiquitin-like molecule interferon-stimulated gene 15 is overexpressed in human prostate cancer
}

\author{
HIROFUMI SATAKE ${ }^{1}$, KENJI TAMURA ${ }^{1}$, MUTSUO FURIHATA $^{2}$, TAKASHI ANCHI ${ }^{1}$, HATSUNE SAKODA $^{1}$, \\ CHIAKI KAWADA ${ }^{1}$, TATSUO IIYAMA ${ }^{3}$, SHINGO ASHIDA ${ }^{1}$ and TARO SHUIN ${ }^{1}$
}

Departments of ${ }^{1}$ Urology, ${ }^{2}$ Pathology and ${ }^{3}$ Immunology, Kochi Medical School, Kochi University, Nankoku 783-8505, Japan

Received August 25, 2009; Accepted October 9, 2009

DOI: $10.3892 /$ or_00000600

\begin{abstract}
To identify molecules to serve as diagnostic markers for high-grade prostate cancer (PC) and targets for novel therapeutic drugs, we investigated the gene expression profiles of high-grade PCs using a cDNA microarray combined with laser microbeam microdissection. We subsequently confirmed that the ubiquitin-like molecule interferon-stimulated gene 15 (ISG15) was expressed exclusively in high-grade PCs with high Gleason scores. Semi-quantitative reverse transcription PCR and immunohistochemical analysis confirmed the overexpression of ISG15, a 165 amino acid interferon-inducible ubiquitin-like protein, specifically in high-grade PCs with high Gleason scores 8-9, while it was not expressed in the normal prostate. Immunohistochemical analysis using anti-ISG15 polyclonal antibody confirmed an elevated expression of ISG15 protein in high-grade PCs as well as low-grade PCs compared with that in normal prostate (NP) epithelium. Knockdown of ISG15 expression by short interfering RNA (siRNA) in a PC cell line resulted in marked attenuation of PC cell survival; concordantly, ISG15 overexpression in a PC cell line promoted PC cell growth, indicating its oncogenic property. These findings suggest that ISG15 is involved in cell growth and survival of PCs and that it could be a potential molecular target for new therapeutics and a diagnostic biomarker for human PCs.
\end{abstract}

\section{Introduction}

Prostate cancer (PC) is the most common malignancy in males and the second leading cause of cancer-related death in Western countries (1) and the incidence of PC has been increasing significantly in most developed countries, probably due to the prevalent western life style and explosion of the aging population $(1,2)$. Screening using serum prostate-specific

Correspondence to: Dr Hirofumi Satake, Department of Urology, Kochi Medical School, Kochi University, Kohasu, Okoh-cho, Nankoku, Kochi 783-8505, Japan

E-mail: jm-satakeh@kochi-u.ac.jp

Key words: interferon-stimulated gene 15, cDNA microarray, prostate cancer antigen (PSA) has lead to a marked improvement in the early detection of prostate cancer and has resulted in an increased population of PC patients with localized disease that can be cured by surgical and radiation therapies $(1,2)$; however, 20 to $30 \%$ of these prostate cancer patients still suffer from disease relapse (3-5).

PC shows relatively good prognosis and hormone ablation therapy or castration is usually effective in most relapsed or advanced PCs; however, once castration-resistant prostate cancer (CRPC) cells emerge or PCs are at an advanced stage with high Gleason scores (GS), there are very limited treatment options for these PC patients, such as docetaxel plus predonisone $(6,7)$, which can still offer a minimal effect on PCs. Hence, it is vital to identify molecular targets for CRPCs or aggressive PCs and develop novel therapies to target those molecules; however, serum PSA levels do not necessarily correlate with GS, the progress level and the prognosis of PCs. Therefore, the development of new biomarkers to predict the prognosis and reflect the progress level and GS accurately is also expected. In this study, to identify molecules to serve as diagnostic markers for high-grade PCs and targets for novel therapeutics, we investigated the gene expression profiles of high-grade PCs using a cDNA microarray combined with laser microbeam microdissection (LMM) to enrich populations of cancer cells to obtain high quality data. Among dozens of genes commonly transactivated in high-grade PC cells, we focused on ISG15 (the ubiquitin-like molecule interferonstimulated gene 15) as an overexpressing gene in high-grade PCs. ISG15 is a 165 amino acid interferon-inducible ubiquitinlike protein that is induced by type I IFNs (8-10). It was the first identified member of the family of ubiquitin-like proteins $(8,11)$, which has grown to include additional protein modifiers with appreciable sequence homology to ubiquitin, such as SUMO, Nedd8 and Fat 10 (12).

Here we validated ISG15 overexpression in clinical PCs with various grades. We also demonstrate its positive involvement in the proliferation or viability of PC cells. Our data could provide new insights into the molecular mechanisms of PC progression and clues to develop new therapeutic strategies or diagnostic biomarkers for PCs.

\section{Materials and methods}

Patients and tissue samples. Tissue samples were obtained after receiving informed consent from 10 high-grade PC 
patients undergoing prostatic needle biopsy before androgen ablation therapy. All samples were embedded in OCT compound (Tissue-Tek) immediately after tissue procurement and stored at $-80^{\circ} \mathrm{C}$ until their use. Histopathologic diagnoses were made by a single pathologist (M.F.) before LMM and H\&E-stained sections from adjacent frozen tissues were prepared to confirm the histological diagnosis. We microdissected cancer cells from these frozen slides by means of LMM (CellCut Laser Microdissection System; Molecular Machines \& Industries AG). Normal prostatic (NP) epithelial cells were also microdissected from 5 benign prostatic hyperplasia patients, where we confirmed no apparent prostate cancer or prostatic intraepithelial neoplasia histopathologically.

cDNA microarray analysis and acquisition of data. Total RNA was extracted from each sample of laser microdissected cells into $350 \mu \mathrm{l}$ RLT lysis buffer (Qiagen). The extracted RNA was treated for $30 \mathrm{~min}$ at room temperature with $30 \mathrm{U}$ DNase I (Roche) in the presence of $1 \mathrm{U}$ RNase inhibitor (Toyobo, Osaka, Japan) to remove any contaminating genomic DNA. After inactivation at $70^{\circ} \mathrm{C}$ for $10 \mathrm{~min}$, the RNA was purified with an RNeasy mini kit (Qiagen) according to the manufacturer's recommendations.

GeneChip array data were compared using Kurabo custom analysis services (Kurabo Industries Ltd., Osaka, Japan). Kurabo Industries Ltd. is an authorized service provider for Affymetrix Japan K.K. (Affymetrix Japan K.K., Tokyo, Japan). Briefly, total RNA was reverse transcripted to cDNA with T7 oligo d(T) primer (Affymetrix, Inc.). The cDNA synthesis product was used in an in vitro transcription reaction containing T7 RNA polymerase. An unlabeled ribonucleotide mix was used in the first cycle of IVT amplification. Unlabeled cRNA was then reverse transcribed in the first-strand cDNA synthesis step of the second cycle using random primers. Subsequently, the T7-Oligo(dT) Promoter Primer was used in second-strand cDNA synthesis to generate a double-stranded cDNA template containing T7 promoter sequences. The resulting doublestranded cDNA was then amplified and labeled using a biotinylated nucleotide analog/ribonucleotide mix in the second IVT reaction. Then, the labeled cRNA products were fragmented, loaded on to GeneChip ${ }^{\circledR}$ Human Genome U133 Plus2.0 array (Affymetrix, Inc.) and hybridized according to the manufacturer's protocol. Streptavidin-phycoerythrin (Molecular Probes) was used as the fluorescent conjugate to detect hybridized target sequences. Raw intensity data from the GeneChip array were annalyzed by GeneChip Operating Software (Affymetrix, Inc.).

Using the gene expression profile analysis of 10 highgrade PCs, we selected several up-regulated genes whose expression ratio was $>5.0$ in $>50 \%$ of informative cases. Finally, to obtain diagnostic markers highly specific to PC and to identify potential molecular targets, we focused on one expressed sequence tag (ISG15).

Cell lines. The human PC cell line 22Rv1, PC3, was obtained from the American Type Culture Collection (ATCC, Rockville, $\mathrm{MD})$. All cell lines were cultured as monolayers in the following medium: DMEM (Sigma-Aldrich) for 22Rv1 and F-12 (Gibco) for PC-3 with 10\% fetal bovine serum and $1 \%$ antibiotic/antimycotic solution (Sigma-Aldrich). Cells were maintained in incubators containing humidified air with $5 \%$ $\mathrm{CO}_{2}$ at $37^{\circ} \mathrm{C}$.

Semi-quantitative RT-PCR. Total RNA was extracted using RNeasy kit (Qiagen) according to the manufacturer's instructions, treated with DNase I (Roche Diagnostic) and reverse transcribed to single-stranded cDNA using a random hexamer or oligo $d(T)_{12-18}$ primer with Superscript reverse transcriptase II (Invitrogen, Carlsbad, CA). We prepared appropriate dilutions of each single-strand cDNA followed by normalizing cDNA content using $\beta$-actin (ACTB) as a quantitative control, demonstrating a PCR reaction using single-strand cDNA as the PCR template. Primer sequences were as follows: $A C T B$ (forward: 5'-TTGGCTTGACTCAG GATTTA-3', reverse: 5'-ATGCTATCACCTCCCCTGTG-3'), ISG15 (forward 5'-TGTCGGTGTCAGAGCTGAAG-3', reverse: 5'-GCCCTTGTTATTCCTCACCA-3'). PCR conditions were as follows: initial denaturation at $95^{\circ} \mathrm{C}$ for 5 min, 23 cycles for $A C T B$ and 30 cycles for $I S G 15$ of denaturation at $95^{\circ} \mathrm{C}$ for $30 \mathrm{sec}$, annealing at $55^{\circ} \mathrm{C}$ for $30 \mathrm{sec}$ and elongation at $72^{\circ} \mathrm{C}$ for $30 \mathrm{sec}$ on a T Gradient Thermocycler (Biometra).

Immunohistochemical analysis. PC tissues were obtained from patients who underwent a prostectomy at Kochi University Medical School with the appropriate informed consent. Immunohistochemical study was carried out using the Ventana automated immunohistochemical system (Discovery; Ventana Medical Systems, Inc., Tucson, AZ). Sections were incubated with a 1:1000 diluted solution of anti-ISG15 pAb (Santa Cruz Biotechnology, Santa Cruz, CA) for 16 min. The automated protocol is based on an indirect biotin-avidin system using a biotinylated universal secondary antibody and diaminobenzidine substrate with hematoxylin counterstaining.

Western blotting. We examined the expression of exogenous ISG15 protein in the PC cell line 22Rv1 using pIRES neo3ISG15-HA-transfected 22Rv1 cells as a positive control and pIRES neo3 vector-22Rv1 cells (Mock) as a negative control. Cell lysates were separated on $15 \%$ SDS-polyacrylamide gels, transferred to nitrocellulose membranes and incubated with anti-HA pAb as the primary antibody. After incubation with goat anti-rabbit IgG-HRP as the secondary antibody (Amersham Biosciences), signals were visualized with an enhanced chemiluminescence kit (Amersham Biosciences). To detect secreted ISG15 protein, ISG15-transfected 22Rv1 cells were maintained in serum-free medium and harvested $48 \mathrm{~h}$ after transfection. ISG15 protein in cell lysates and condensed culture media were also detected by ISG15-pAb and goat anti-rabbit IgG-HRP. B-actin pAb (1:5,000 dilution) served as a loading control for proteins (clone AC-15, Sigma-Aldrich, St. Louis, MO).

Construction of short hairpin RNA (shRNA)-expressing vectors and cell viability assay. To investigate the biological function of ISG15 in PC cells, we used pBAsi-h6 Neo vector (Takara Bio Inc.) for the expression of short-hairpin RNA (shRNA) against a target gene. Plasmids designed to express shRNA were prepared by cloning double-stranded oligonucleotides into pBAsi-h6 Neo vector. The oligonucleotide sequences of 
target sequences for ISG15 were as follows: sense strand sequence for si1: 5'-GCAGATCACCCAGAAGATT-3', si2: 5'-GCATCCTGGTGAGGAATAA-3' and negative control scramble siRNA(siSC): 5'-GAAGCAGCACGACTTCTTC-3' as described previously (13). 22Rv1 cells $\left(2 \times 10^{6}\right)$ which expressed ISG15 at a high level, were seeded in $10-\mathrm{cm}$ dishes, transfected with pBA-ISG15 (si1,2), or pBA-siSC using FuGENE6 (Roche Diagnostics) according to the manufacturer's instructions and then cultured in appropriate medium containing $800 \mu \mathrm{g} / \mathrm{ml}$ Geneticin (Sigma-Aldrich) for 14 days. The cells were fixed with $100 \%$ methanol and stained with $0.1 \%$ crystal violet- $\mathrm{H}_{2} \mathrm{O}$ for colony formation assay. In the MTT assay, cell viability was measured using the Cell-counting kit-8 (Dojindo, Kumamoto, Japan) 10 days after transfection. Absorbance was measured at $490 \mathrm{~nm}$ and at $630 \mathrm{~nm}$ as a reference, with a microplate reader (Molecular Devices THERMOmax). Preliminarily, the knockdown effects of these shRNA expression vectors on endogenous ISG15 expression were validated 7 days after transfection by RT-PCR using the primers for semi-quantitative RT-PCR.

Generation of ISG15 overexpressing cells and in vitro growth assay. Full-length human ISG15 cDNA (accession no. NM005101) was amplified using primers that were designed to contain HA-tag sequences at the $\mathrm{COOH}$ terminus and cloned into the pIRESneo3 vector (Clontech). Human PC cell line $22 \mathrm{Rv} 1$ cells were seeded into $100-\mathrm{mm}$ dish $\left(5 \times 10^{5}\right.$ cells per dish) and transfected with $6 \mu \mathrm{g}$ pIRESneo3 empty vector or pIRESneo3-ISG15-HA expression vector using FuGENE6 reagent (Roche) according to the manufacturer's instructions. Cells were selected with appropriate medium containing $400 \mu \mathrm{g} / \mathrm{ml}$ Geneticin (Sigma-Aldrich) for 14 days when discrete colonies were isolated. All clones were maintained in selective medium. Each clone was assayed for ISG15 protein expression by Western blot analysis using anti-HA tag antibody (Roche). Proliferation of 22Rv1 cells that stably expressed ISG15 (22Rv1-ISG15) or those transfected with pIRESneo3 empty vector (22Rv1-mock clone mixture) were examined using the Cell-counting kit-8 (Dojindo). Each of 22Rv1-ISG15 and 22Rv1-mock cells was seeded at a concentration of $3 \times 10^{3}$ cells per well using 48 -well plates. The assay was performed every $48 \mathrm{~h}$ for 9 days, according to the manufacturer's instructions.

\section{Results}

ISG15 overexpression in PC cells. For this study, we collected 10 frozen specimens from high-grade PCs with high PSA levels and high GS in clinically using prostatic needle biopsy. All needle biopsy specimens were at clinical stages T2 to T4 with or without N1 and M1 and their GS were 8-9 (Table I). Moreover, all 10 patients had not received androgen ablation therapy. Through pathologic evaluation by H\&E staining or after evaluation of their RNA quality, RNA from 10 patients was available for further microarray. Simultaneously, NP epithelial cells were also microdissected from five non-prostate cancer patients. These NP cells from five males were used as a normal mixture control for our cDNA microarray analysis. We successfully microdissected high-grade PC cells and NP cells from each clinical sample to exclude the contamination
Table I. Clinical and pathological characteristics in 10 highgrade PC patients used for LMM and cDNA microarray analysis.

\begin{tabular}{rrrcl} 
Pt. No. & Age & PSA (ng/ml) & Gleason score & Clinical stage \\
\hline 1 & 83 & 5477 & 9 & T4N0M1 \\
2 & 81 & 4427 & 9 & T4N1M1 \\
3 & 85 & 1900 & 9 & T2bN1M1 \\
4 & 75 & 630 & 9 & T3aN0M1 \\
5 & 74 & 334 & 9 & T3bN0M1 \\
6 & 57 & 311 & 9 & T4N1M1 \\
7 & 62 & 1000 & 8 & T3bN1M1 \\
8 & 81 & 275 & 9 & T3bN0M0 \\
9 & 72 & 80 & 9 & T3aN0M0 \\
10 & 79 & 234 & 8 & T3aN1M0 \\
\hline
\end{tabular}

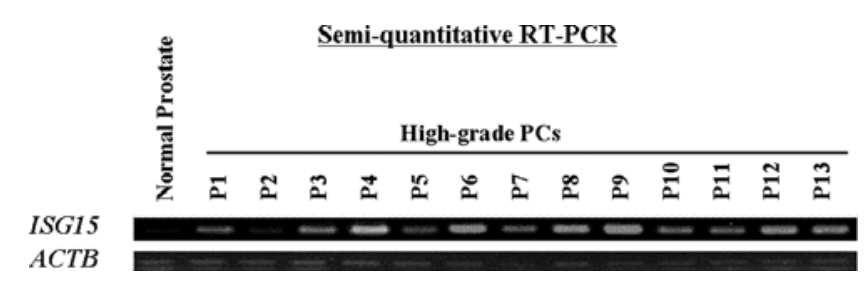

Figure 1. ISG15 overexpression in high-grade PC cells. Semi-quantitative RT-PCR confirmed that ISG15 was overexpressed in microdissected highgrade PC cells with high PSA levels and high GS, compared with normal prostatic epithelial (NP) cells, which were also microdissected. ACTB was used to quantify the cDNA contents.

of stromal cells. Among a number of transactivated genes in high-grade PC cells compared with normal prostate epithelial cells (NP), we focused on ISG15 in this study. Semi-quantitative RT-PCR confirmed the elevated expression of ISG15 in 13 microdissected high-grade PC cells with high PSA levels and high Gleason scores, compared with NP cells (Fig. 1).

Immunohistochemical analysis of ISG15 in clinical PCs. To validate the overexpression of ISG15 protein in clinical PC cells, we performed immunohistochemical analysis on clinical PC tissues with various GS using polyclonal antibody specific to human ISG15. Immunohistochemical analysis confirmed the highly elevated expression of ISG15 in prostate cancer cells compared with in normal prostate epithelium. A strong immunochemical signal for ISG15 was detected predominantly in the cytoplasm, partially in the nucleus of PC cells (Fig. 2). We analyzed the clinical or pathologic significance of ISG15 in 57 PC tissues; ISG15 expression was not associated with GS of PCs.

Knockdown of ISG15 expression by shRNA attenuated PC cell growth. To examine the biological roles of ISG15 overexpression in PC cells, we constructed two vectors designed to express shRNA specifically to ISG15 and transfected them into 22Rv1, which is an androgen receptor (AR)-positive PC cell line (Fig. 3A). Among the two shRNA expression 


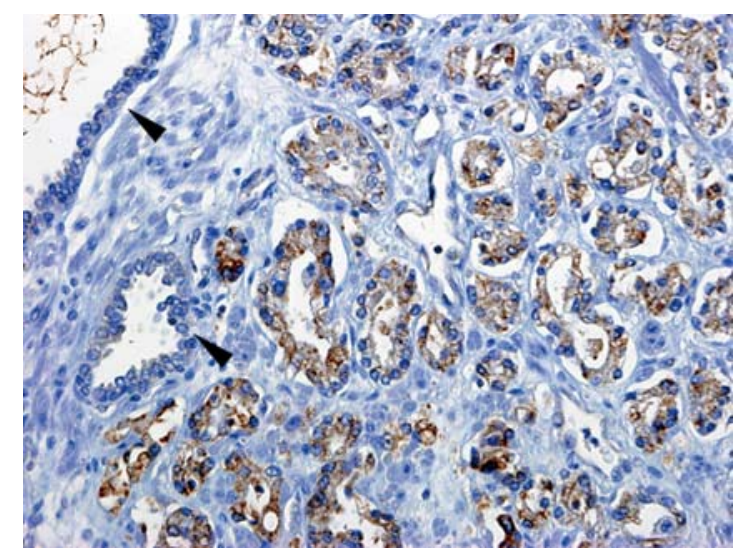

Figure 2. Immunohistochemical analysis of ISG15 in PC tissues. Immunohistochemical analysis using anti-ISG15 polyclonal antibody confirmed the elevated expression of ISG15 protein in PC cells compared with in normal prostate epithelium. A strong immunochemical signal for ISG15 was detected predominantly in the cytoplasm of cancer cells, partially in the nucleus. Adjacent normal prostate epithelium (arrowhead) in the same patient revealed a very weak or no signal for ISG15.

vectors, si1 and si2 showed a significant knockdown effect on endogenous $I S G 15$ transcript and this transfection resulted in a reducted number of colonies as well as of viable cells measured by MTT assay for 22Rv1 cells. On the other hand, the transfection of a negative control (siSC) had no or little knockdown effect on ISG15 expression and did not affect the cell viability of 22Rv1 cells (Fig. 3B and C). These findings indicated that ISG15 overexpression played important roles in PC cell growth or survival. Similar results were obtained in PC-3, which is an AR-negative PC cell line (data not shown).
ISG15 overexpression promoted cancer cell growth. To further investigate the potential oncogenic function of ISG15, we established stable transformants from 22Rv1 cells, in which exogenous ISG15 was expressed constitutively. We also prepared control 22Rv1 cells transfected with empty vector (Mock) and compared their proliferation. Western blot analysis confirmed a high level of exogenous ISG15 expression in the stable clone. MTT assay showed that the ISG15 overexpressing clone grew more rapidly than the $22 \mathrm{Rv} 1$-mock clone $\left({ }^{*} \mathrm{P}<0.01\right.$, ${ }^{* *} \mathrm{P}<0.05$, Student's t-test), indicating that ISG15 overexpression promoted PC cell growth (Fig. 4A and B).

Exogenous expression of ISG15 protein. Expression of endogenous and exogenous ISG15 protein in PC cell line 22Rv1 were examined by Western blot analysis using cell lysates (CL) and culture medium (CM).

22Rv1 cells were transiently transfected with ISG15expressing vector (pIRES neo3/ISG15-HA vector) and a negative control vector (MOCK, pIRES neo3 empty vector) using FuGENE6 reagent according to the manufacturer's instructions. Transfected 22Rv1 cells were maintained in serum-free medium and harvested $30 \mathrm{~h}$ after transfection. As a result of Western blot analysis, the secretion of ISG15 protein into the culture medium was detected (Fig. 5).

\section{Discussion}

In this study, we identified ISG15 as a novel target molecule for therapy development or as a biomarker of PCs. ISG15 is a $15-\mathrm{kDa}$ protein that is induced by viral infection through the Janus kinase/signal transducer and an activator of the transcription (JAK/STAT) signaling pathway $(14,15)$. ISG15
(A)RT-PCR

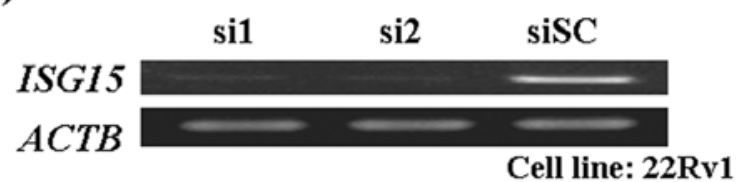

(B)colony formation assay

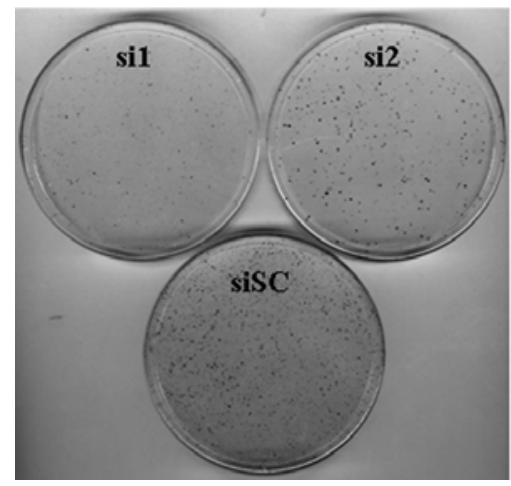

Cell line: 22Rv1 pBAsi-U6 / ISG15
(C)MTT assay

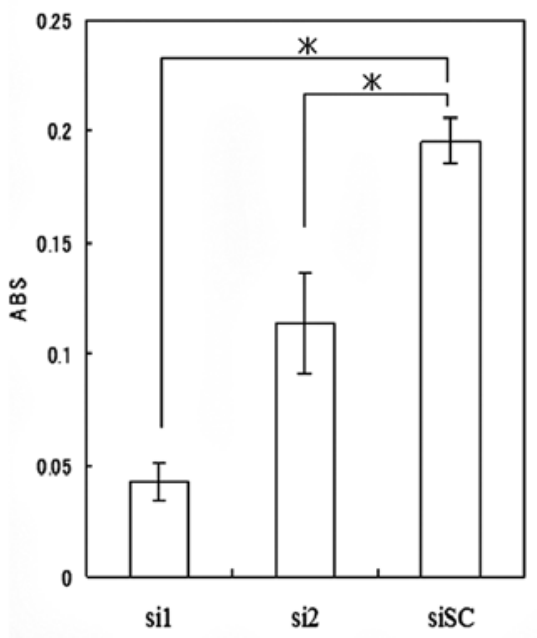

Figure 3. Knockdown of ISG15 expression by siRNA attenuated PC cell viability. (A) Knockdown effect of siRNA on ISG15 in 22 Rv1 cells. Semi-quantitative RT-PCR was performed using cells transfected with each shRNA-expressing vector to ISG15 (si1, 2) as well as a negative control vector (siSC). B-actin (ACTB) was used to quantify RNA. (B) Colony formation assay of 22Rv1 cells transfected with each of the indicated shRNA-expressing vectors to ISG15 $($ si1, 2) and a negative control vector (siSC). Cells were visualized with $0.1 \%$ crystal violet staining after 14 -day incubation with Geneticin. (C) MTT assay of each of the 22Rv1 cells transfected with indicated the shRNA-expressing vectors to ISG15 (si1, 2) and a negative control vector (siSC). Each average is plotted with error bars indicating SD (standard deviation) after 10-day incubation with Geneticin. ABS on Y-axis means absorbance at $490 \mathrm{~nm}$ and at $630 \mathrm{~nm}$ as a reference, measured with a microplate reader. These experiments were carried out in triplicate $\left({ }^{*} \mathrm{P}<0.01\right.$, Student's t-test). 
(A) ISG15; stable transformant

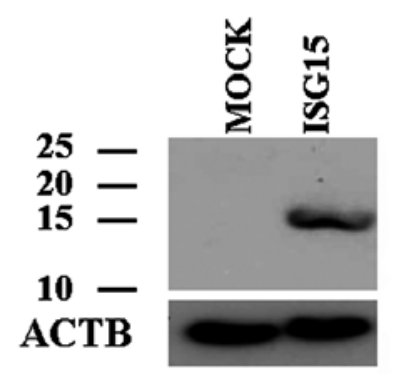

$1^{\text {st }}$ Ab: HA tag Ab 1/2000

Cell line: 22Rv1 pIRES / ISG15-HA
(B)

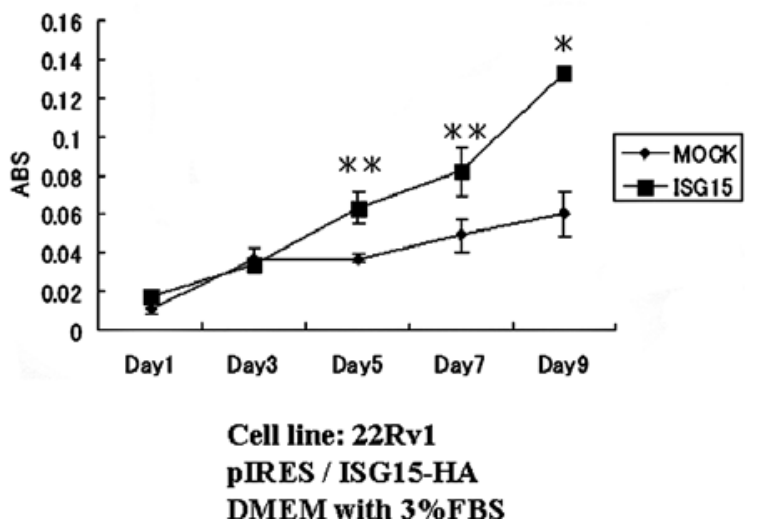

Figure 4. ISG15 overexpression promotes cancer cell growth. (A) Western blot analysis with anti-HA-tag antibody validated the constitutive expression of exogenous ISG15 in stable transformants. Mock was the 22Rv1-mock clone. (B) In vitro growth curve by MTT assay showed that stable transformants grew more rapidly than the 22Rv1-mock clone $\left({ }^{*} \mathrm{P}<0.01,{ }^{* *} \mathrm{P}<0.05\right.$, Student's t-test). ABS on Y-axis means absorbance at $490 \mathrm{~nm}$ and at $630 \mathrm{~nm}$ as a reference, measured with a microplate reader.

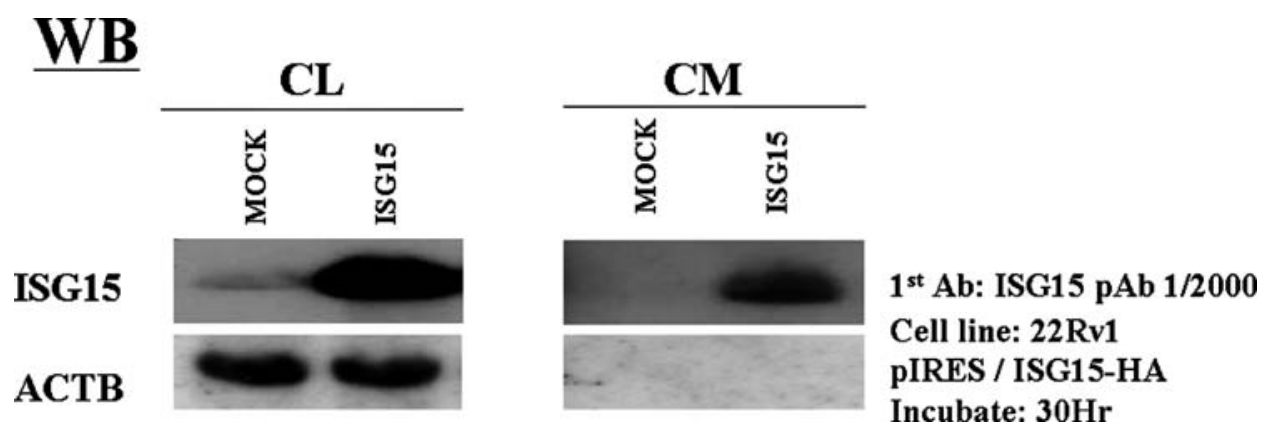

Figure 5. Exogenous expression of ISG15 protein. Expression of endogenous and exogenous ISG15 protein in PC cell line $22 \mathrm{Rv} 1$ were examined by Western blot analysis using cell lysates (CL) and culture medium (CM). 22Rv1 cells were transiently transfected with ISG15-expressing vector (pIRES neo3/ISG15-HA vector) and a negative control vector (MOCK, pIRES neo3 empty vector) using FuGENE6 reagent according to the manufacturer's instructions. Transfected 22Rv1 cells were maintained in serum-free medium and harvested $30 \mathrm{~h}$ after transfection.

is conjugated to intracellular proteins by a mechanism called ISGylation, which is similar to ubiquitin (ubiquitination). ISGylation is also composed of activating E1, conjugating E2 and ligation E3 enzymes as well as protein ubiquitination. ISGylated proteins with different functions have been identified; however, how ISG15 and ISGylation affect their cellular distribution and function is yet not clear (16-18). So far, their physiological or pathological functions in human cancers have not been clearly elucidated.

Our immunohistochemical study using anti-ISG15 polyclonal antibody clearly indicated ISG15 overexpression in PC cells. Knockdown of ISG15 expression by siRNA resulted in marked reduction of PC cell numbers. Concordantly, exogenous ISG15 expression in transfected cells promoted PC cell growth. These findings implied the positive involvement of ISG15 in the proliferation or viability of PC cells and could provide new insights into the molecular mechanisms of PC progression. Furthermore, we demonstrated the secretion of ISG15 protein into culture medium. It is possible that ISG15 could be a new diagnostic biomarker for PCs.

It is thought that ISG15 may play a role in the antivial innate immune response. Futhermore, increased expression of ISG15 and ISGylated protein have been shown in human cancer cell lines (19). In addition, in the current study, it was reported that the ISGylation system controled AR mRNA and protein expression and AR played a crucial role in the modulation of prostate cell proliferation and was involved in the development and progression of prostate cancer (20). In our study, we also showed a positive association between the expression of ISG15 and PC cell growth or survival. Interestingly, these results were obtained in AR-negative PC cells as well as AR-positive PC cells. These findings suggested that ISG15 overexpression might be related to cell growth and/or survival in not only androgen-dependent PCs but also androgen-independent PCs. Therefore, ISGylation system also might regulate AR bypass pathways or other pathways. We have tried to identify ISGylated proteins in PC cells, however, we have not found any yet. It is possible that ISG15 by itself can function as a signaling pathway related to PC cell growth and/or survival.

In summary, the ISG15 and/or ISGylation system might be essential in the cell viability of PCs, although the detailed mechanisms in PCs remain unknown and should be defined by further investigations. The detection of ISG15 and its inhibition could provide a novel promising approach for a biomarker or molecular treatment of PCs. 


\section{Acknowledgements}

We thank Dr Yoshihiro Kamei, Fumito Komatsu, Masanobu Tanimura, Hironobu Watanabe, Shinichi Kataoka, Masaharu Yasuda, Koji Sawada, Masakazu Chikazawa for providing the tissue samples from prostate cancer patients.

\section{References}

1. Gronberg H: Prostate cancer epidemiology. Lancet 361: 859-864, 2003.

2. Hsing AW and Devesa SS: Trends and patterns of prostate cancer: what do they suggest? Epidemiol Rev 23: 3-13, 2001.

3. Feldman BJ and Feldman D: The development of androgenindependent prostate cancer. Nat Rev Cancer 1: 34-45, 2001.

4. Scher HI and Sawyers CL: Biology of progressive, castrationresistant prostate cancer: directed therapies targeting the androgen-receptor signaling axis. J Clin Oncol 23: 8253-8261, 2006.

5. Han M, Partin AW, Piantadosi S, Epstein JI and Walsh PC: Era specific biochemical recurrence-free survival following radical prostatectomy for clinically localized prostate cancer. J Urol 166 416-419, 2001

6. Tannock IF, de Wit R, Berry WR, et al: Docetaxel plus predonisone or mitoxantrone plus predonisone for advanced prostate cancer. N Engl J Med 351: 1502-1512, 2004.

7. Petrylak DP, Tangen CM, Hussain MH, et al: Docetaxel and estramustine compared with mitoxantrone and predonisone for advanced refractory prostate cancer. N Engl J Med 351: 1513-1520, 2004.

8. Haas AL, Ahrens P, Bright PM and Ankel H: Interferon induces a 15-kilodalton protein exhibiting marked homology to ubiquitin. J Biol Chem 262: 11315-11323, 1987.

9. Farrell PJ, Broeze RJ and Lengyel P: Accumulation of an mRNA and protein in interferon-treated Ehrlich ascites tumour cells. Nature 279: 523-525, 1979.
10. Korant BD, Blomstrom DC, Jonak GJ and Knight EJ: Interferoninduced proteins. Purification and characterization of a 15,000dalton protein from human and bovine cells induced by interferon. J Biol Chem 259: 14835-14839, 1984.

11. Loeb KR and Haas AL: The interferon-inducible 15-kDa ubiquitin homolog conjugates to intracellular proteins. J Biol Chem 267: 7806-7813, 1992.

12. Hochstrasser M: Evolution and function of ubiquitin-like proteinconjugation systems. Nat Cell Biol 2: E153-E157, 2000.

13. Uemura M, Tamura K, Chung S, et al: Movel $5 \alpha$ steroid reductase (SRD5A3, type-3) is overexpressed in hormone-refractory prostate cancer. Cancer Sci 99: 81-86, 2008.

14. Stark GR, Kerr IM, Williams BR, Silverman RH and Schreiber RD: How cells respond to interferons. Annu Rev Biochem 67: 227-264, 1998.

15. de Veer MJ, Holko M, Frevel M, et al: Functional classification of interferon-stimulated genes identified using microarrays. J Leukoc Biol 69: 912-920, 2001.

16. Malakhov MP, Kim KI, Malakhova OA, Jacobs BS, Borden EC and Zhang DE: High-throughput immunoblotting. Ubiquitinlike protein ISG15 modifies key regulators of signal transduction. J Biol Chem 278: 16608-16613, 2003.

17. Giannakopoulos NV, Luo JK, Papov V, et al: Proteomic identification of proteins conjugated to ISG15 in mouse and human cells. Biochem Biophys Res Commun 336: 496-506, 2005.

18. Zhao C, Denison C, Huibregtse JM, Gygi S and Kruq RM: Human ISG15 conjugation targets both IFNinduced and constitutively expressed proteins functioning in diverse cellular pathways. Proc Natl Acad Sci USA 102: 10200-10205, 2005.

19. Desai SD, Haas AL, Wood LM, et al: Elevated expression of ISG15 in tumor cells interferes with the ubiquitin/26S proteasome pathway. Cancer Res 66: 921-928, 2006.

20. Kiessling A, Hogrefe C, Erb S, Bobach S, Fuessel S, Wessjohann L and Seliger B: Expression, regulation and function of the ISGylation system in prostate cancer. Oncogene 28: 2606-2620, 2009. 